\title{
Correlated ion hopping in single-crystal yttria-stabilized zirconia
}

\author{
C. León, M. L. Lucía, and J. Santamaría \\ Departamento de Física Aplicada III, Facultad de Ciencias Físicas, Universidad Camplutense de Madrid, \\ Avda. Complutense s/n. 28040 Madrid, Spain
}

(Received 29 July 1996)

\begin{abstract}
A study of the effect of correlated ion motion on the electrical conductivity relaxation in single-crystalline yttria-stabilized zirconia is presented. Complex admittance in the radio frequency range show power-law dependencies in the real part of the conductivity at high frequencies of the form $\omega^{n}$ and asymmetric electric modulus plots as a result of correlations. An analysis of the frequency dependence of the electric modulus is conducted to obtain time decay functions of the form $\exp \left[-(t / \tau)^{\beta}\right]$ from an analytical distribution of relaxation times. Correlation times, and parameters $n$ and $\beta$ characterizing the relaxation in time and frequency domains are compared to show the equivalence of time and frequency representations. The common origin of ac and $\mathrm{dc}$ processes is discussed in view of the frequency dependence of the complex conductivity. From a macroscopic activation energy for ion motion $E=1.16 \mathrm{eV}$ and a $\beta$ value of 0.43 , a single-ion microscopic activation energy $E_{a}=0.5 \mathrm{eV}$ is obtained as $\beta E$ according to Ngai's coupling model. The microscopic activation energy is related to the association energy of oxygen vacancies. [S0163-1829(97)07301-3]
\end{abstract}

\section{INTRODUCTION}

The high oxygen conductivity $\left(0.1 \Omega^{-1} \mathrm{~cm}^{-1}\right)$ of yttriastabilized zirconia $\left(\mathrm{ZrO}_{2}: \mathrm{Y}_{2} \mathrm{O}_{3}\right)$ at high temperatures $\left(1000^{\circ} \mathrm{C}\right)$ has been known for many years ${ }^{1}$ and has made this material one of the most extensively studied fast anionic conductors for its applications in fuel cells and oxygen sensing devices. Doping with $\mathrm{Y}_{2} \mathrm{O}_{3}$ is known to stabilize the cubic fluorite structure of $\mathrm{ZrO}_{2}$ and to supply the oxygen vacancies responsible for the ionic conduction. However there remain open questions regarding the conduction process. Oxygen vacancies in yttria-stabilized zirconia (YSZ) are known to form clusters at temperatures below $900{ }^{\circ} \mathrm{C}$, giving rise to the so-called associated regime. This process adds an association energy to the migration energy found at higher temperatures, so that two slopes are observed in conductivity vs temperature experiments extending up to high enough temperatures. A mechanism has been proposed recently which accounts for the temperature dependence of the conductivity. $^{2}$ It is based on a noncorrelated randomly distributed oxygen vacancy hopping at high temperatures. An association mechanism of oxygen vacancies is proposed, such that below $673 \mathrm{~K}$ all the vacancies are assumed to form clusters. It is below this temperature where one would expect correlation effects to be dominant. Although correlation effects have been extensively investigated in disordered electrolytes, and are known to be responsible for non-Debye relaxation of the conductivity, up to our knowledge, there is a lack of experimental work devoted to the study of the conductivity relaxation in crystalline solids at low temperatures, and specially in YSZ. We present a study of the electrical conductivity relaxation (ECR) from complex admittance measurements.

Two alternative formalisms are usually employed in the analysis of the ECR, the conductivity formalism in the frequency domain, and the electric modulus formalism which uses electric modulus data to obtain the decay function in the time domain.
A sublinear frequency dispersive ac conductivity has been frequently observed at high frequencies, such that the real part of the conductivity $\sigma^{\prime}(\omega)$ can be expressed as

$$
\sigma^{\prime}(\omega)=\sigma_{0}+A \omega^{\prime \prime}
$$

where $\sigma_{0}$ is the dc conductivity, $A$ is a temperature dependent parameter and $n$ is a fractional exponent between 0 and 1 , which has been interpreted to appear as a result of manybody interactions among charge carriers. This behavior, termed universal dynamic response, has been widely observed in highly disordered materials like ionically conducting glasses, conducting polymers, and amorphous semiconductors and also in doped crystalline solids. ${ }^{3}$

An alternative representation of experimental data has been made in terms of the time decay $[\Phi(t)]$ of the electric field at constant displacement vector. Kohlrausch-WilliamsWatts (KWW), "stretched" exponential decay functions of the form

$$
\Phi(t)=\exp \left[-\left(t / \tau^{*}\right)^{\beta}\right]
$$

have been frequently observed in glassy systems, where $\tau^{*}$ is a temperature activated relaxation time and $0<\beta<1$. The electric modulus is then expressed as

$$
M^{*}(\omega)=M_{\infty}\left(1-\int_{0}^{\infty}(-d \Phi / d t) e^{-j \omega t} d t\right),
$$

where $M_{\infty}$ is the inverse of the high-frequency dielectric permittivity. Some models ${ }^{5,6}$ have been proposed to explain the non-Debye behavior of the conductivity relaxation in structurally disordered solids, suggesting that a slowing of the relaxation process occurs as a result of correlated ion hopping. The stretched exponential parameter $\beta$ may represent an index of correlation of ionic motion, so that one would expect $\beta$ to be close to zero for strongly correlated systems and 1 for random Debye-like hops. ${ }^{7}$ 


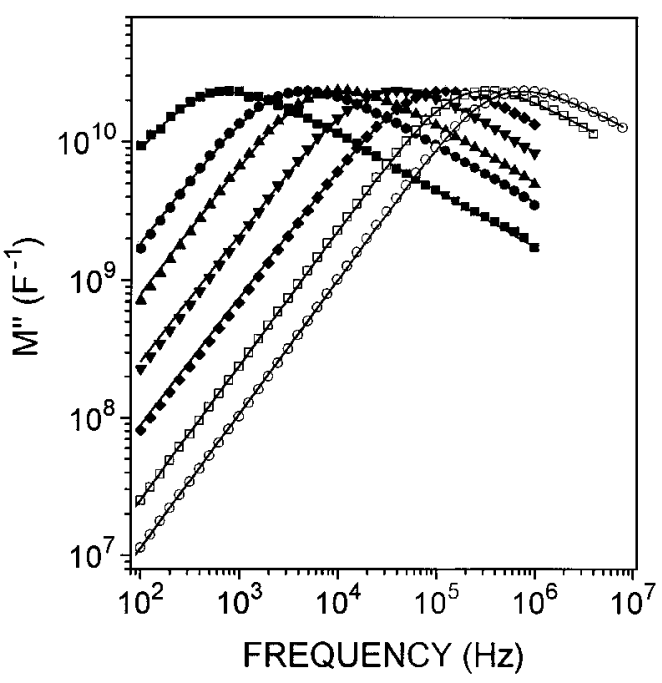

FIG. 1. Imaginary part of the electric modulus vs frequency presented in a double-logarithmic scale at $480 \mathrm{~K}(\boldsymbol{\square}), 514 \mathrm{~K}$ $(\bullet), 532 \mathrm{~K}(\boldsymbol{\Delta}), 560 \mathrm{~K}(\boldsymbol{\nabla}), 587 \mathrm{~K}(\bullet), 615 \mathrm{~K}(\square)$, and $639 \mathrm{~K}$ (O). Solid lines are fits of $M^{\prime \prime}(\omega)$ using $\mathrm{HN}$ functions according to the expression: $1-M^{*}(\omega) / M_{\infty}=F_{\mathrm{HN}}^{*}(\omega)$.

The connection between frequency and time domain representations of the electrical conductivity relaxation has been investigated in single-crystal YSZ. A single-ion microscopic activation energy has been obtained from the activation energy of the relaxation time and the $\beta$ parameter following Ngai's coupling model. We have related this microscopic activation energy to the association energy of oxygen vacancies.

\section{EXPERIMENT}

Samples were single crystals $(100)$ oriented $10 \times 5 \times 1 \mathrm{~mm}$ supplied by Superconductive Components Inc. with composition $\mathrm{ZrO}_{2}-9.5 \mathrm{~mol} \% \mathrm{Y}_{2} \mathrm{O}_{3}$. Contacts were evaporated gold spots on both sides of the crystal.

Complex admittance was measured using a HP4284A precision LCR meter in the frequency range $20 \mathrm{~Hz}-1 \mathrm{MHz}$ and a HP4192A impedance analyzer in the range $5 \mathrm{~Hz}$ to 10 $\mathrm{MHz}$. The temperature was varied between 50 and $650 \mathrm{~K}$. Complex admittance measurements have been conducted at temperatures below $650 \mathrm{~K}$ to avoid the nonlinear electrode polarization effects. Measurements were conducted under high-purity $\mathrm{N}_{2}$ flow or under vacuum to avoid water condensation on the samples.

\section{RESULTS AND DISCUSSION}

Figure 1 shows the imaginary part of the electric modulus vs measuring frequency at various temperatures. A doublelogarithmic scale has been used to bring out the power-law behaviors at both sides of the maximum, showing the strong non-Debye character of the relaxation process.

The time decay function $\Phi(t)$, can be obtained from modulus data according to expression (3) of the Introduction, which relates electric modulus to the Fourier transform of the time derivative of the decay function. If we denote $\Phi^{*}(\omega)$ and $\dot{\Phi}^{*}(\dot{\omega})$ the Fourier transforms of $\Phi(t)$ and $d \Phi(t) / d t$ respectively, this expression can be written as

$$
M^{*}(\omega) / M_{\infty}=1+\dot{\Phi} *(\omega)
$$

The decay function $\Phi(t)$ can thus be calculated Fouriertransforming measured data in the frequency domain. Then, on the base of a KWW dependence of the decay function, the parameters $\beta$ and $\tau^{*}$ characterizing the relaxation mechanism can be obtained. However, numerical errors are known to arise in the calculation of the Fourier transform of a discrete data set extending over a finite frequency window. This problem is usually solved using a discrete distribution of relaxation times, whose determination involves fitting the frequency response with a large number of parameters, as a mathematical tool to do the numerical integration. When using this procedure, modulus plots are customarily presented in a linear scale vs frequency which obscures asymptotic power-law behaviors at low and high frequencies. We propose an alternative method, which based on the analysis of the frequency response of modulus data, permits using an analytical distribution of relaxation times to obtain the time decay function by numerical integration.

The asymmetric power-law behavior of the modulus data at low and high frequencies, presented in Fig. 1 suggests that conductivity relaxation may be represented by a HavriliakNegami (HN) relaxation function $\left[F_{\mathrm{HN}}^{*}(\omega)\right]$. The HN function was first introduced by Havriliak and $\mathrm{Negami}^{8}$ to describe the relaxation of glass-forming polymeric systems and it has been frequently used with this purpose afterwards. ${ }^{9}$ It takes the form

$$
F_{\mathrm{HN}}^{*}(\omega)=\frac{1}{\left[1+\left(j \omega \tau_{\mathrm{HN}}\right)^{\alpha}\right]^{\gamma}},
$$

where $\tau_{\mathrm{HN}}$ defines a characteristic relaxation time and $\alpha$ and $\gamma$ are fractional exponents comprised between zero and one. We have found HN function to account very well for the measured conductivity relaxation in YSZ, i.e., $1-M^{*}(\omega) / M_{\infty}=F_{\mathrm{HN}}^{*}(\omega)$. Lines in Fig. 1 are fits of modulus plots to $\mathrm{HN}$ functions according to expression (4).

The parameters $\tau_{\mathrm{HN}}, \alpha$, and $\gamma$ best fitting experimental data can be used to construct an analytical distribution of relaxation times $[\rho(\tau)]$ according to the following expressions:

$$
\rho(\tau)=\frac{1}{\pi} \frac{\left(\tau / \tau_{\mathrm{HN}}\right)^{\alpha \gamma} \sin (\gamma \theta)}{\left[\left(\tau / \tau_{\mathrm{HN}}\right)^{2 \alpha}+2\left(\tau / \tau_{\mathrm{HN}}\right)^{\alpha} \cos (\alpha \pi)+1\right]^{\gamma / 2}},
$$

where $\theta$ is

$$
\theta=\arctan \left|\frac{\sin (\alpha \pi)}{\left(\tau / \tau_{\mathrm{HN}}\right)^{\alpha}+\cos (\alpha \pi)}\right| .
$$

Since according to expression (3) we can relate the electric modulus to $F_{\mathrm{HN}}^{*}(\omega)$ through

$$
1-M^{*}(\omega) / M_{\infty}=F_{\mathrm{HN}}^{*}(\omega)=-\dot{\Phi}^{*}(\omega),
$$

we can express the frequency dependence of the Fourier transform of the time derivative of the decay function as a 


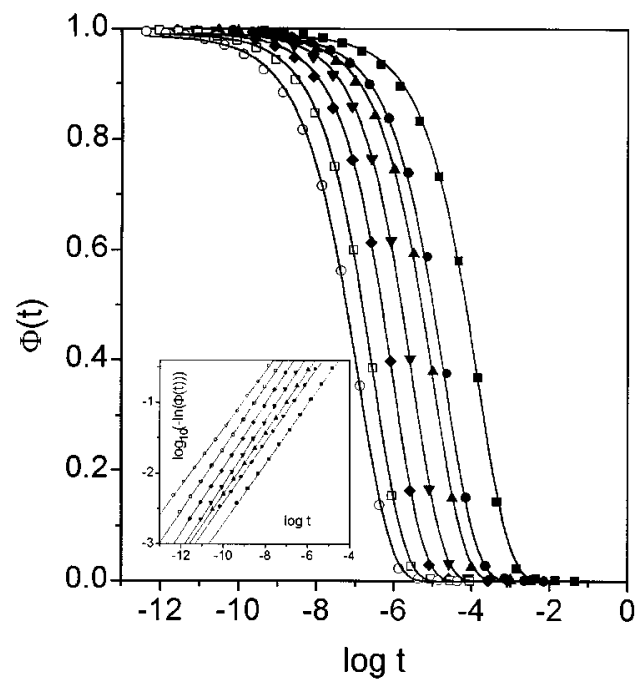

FIG. 2. Time decay functions $\Phi(t)$ obtained at $480 \mathrm{~K}(\boldsymbol{\square}), 514$ $\mathrm{K}(\bullet), 532 \mathrm{~K}(\boldsymbol{\Delta}), 560 \mathrm{~K}(\boldsymbol{\nabla}), 587 \mathrm{~K}(\bullet), 615 \mathrm{~K}(\square)$, and 639 $\mathrm{K}(\bigcirc)$. Solid lines are fits to KWW functions. The insets show log $\{-\ln [\Phi(t)]\}$ versus $\log t$ plots, and lines are linear fits.

superposition of Debye-like processes using the same distribution of relaxation times obtained from the fitting to an $\mathrm{HN}$ function:

$$
-\dot{\Phi} *(\omega)=\int_{0}^{\infty} \frac{\rho(\tau)}{1+j \omega \tau} d \tau
$$

So that in the time domain the following expression holds:

$$
\Phi(t)=\int_{0}^{\infty} \rho(\tau) e^{-t / \tau} d \tau,
$$

which allows determining the time decay function by numerical integration. The infinite range of integration does not pose serious problems because the contribution due to small and large values of $\tau$, compared to $\tau_{\mathrm{HN}}$, is negligible.

Results of the so-obtained decay functions are presented in Fig. 2 for different temperatures. Lines are fits to KWW functions of the form

$$
\Phi(t)=\exp \left[-\left(t / \tau^{*}\right)^{\beta}\right]
$$

showing that stretched exponential relaxation functions satisfactorily account for the relaxation process. The inset of the figure shows the corresponding linear $\log \{-\ln [\Phi(t)]\}$ versus $\log t$ plots from which parameters $\beta$ and $\tau^{*}$ have been obtained by a linear least-squares fitting. Nearly temperature independent $\beta$ values, $0.43 \pm 0.03$, have been obtained over the temperature range where well resolved modulus peaks were obtained. Concerning the relaxation time $\tau^{*}$, it has been proposed ${ }^{10}$ that an average relaxation time, $\langle\tau\rangle$, can be defined in terms of the integrated area of the KWW function as

$$
\langle\tau\rangle=\int_{0}^{\infty} \Phi(t) d t=\frac{\Gamma(1 / \beta) \tau^{*}}{\beta},
$$

where $\Gamma$ refers to the Euler gamma function. $\langle\tau\rangle$ is related to the dc conductivity according to the well known expression

$$
\langle\tau\rangle=\varepsilon_{\infty} / \sigma_{0},
$$

where $\varepsilon_{\infty}$ is the real part of the permittivity at high frequencies, which takes a value of $2.8 \pm 0.2 \times 10^{-10} \mathrm{~F} / \mathrm{m}$, practically temperature independent. The ionic conductivity obtained in this way was thermally activated with $1.16 \mathrm{eV}$ and in good agreement with the values reported by other authors. ${ }^{2,11}$

While the modulus representation is preferred to obtain the time decay function $\Phi(t)$, the frequency dependence of the conductivity is usually employed to analyze the relaxation process in the frequency domain. Some authors use expression (1) to fit their data of the real part of the conductivity.

The real part of the conductivity vs frequency is presented in Fig. 3(a) at different temperatures. A power-law dependence is observed for high-frequencies and a crossover to a frequency independent dc regime occurs at low frequencies. Power laws are also observed in the real part of the permittivity. Figure 3(b) shows the frequency dependence of $\varepsilon^{\prime}(\omega)$ from which the high-frequency permittivity, $\varepsilon_{\infty}$, has been subtracted, at different temperatures. According to Kramers-Kronig relations, power-law dependencies of conductivity and permittivity at high frequencies imply a complex power-law dependence of the form $(j \omega)^{n}$ for the complex conductivity, i.e.,

$$
\sigma^{*}(\omega)=\sigma_{0}+A(j \omega)^{n}+j \omega \varepsilon_{\infty} .
$$

Defining a crossover frequency $\omega_{p}$ the prefactor $A$ can be split in two terms such that expression (1) can be rewritten as

$$
\sigma^{*}(\omega)=\sigma_{0}+\sigma_{0}\left(j \omega / \omega_{p}\right)^{n}+j \omega \varepsilon_{\infty} .
$$

Consequently, the real and imaginary parts of the conductivity can be expressed as

$$
\begin{gathered}
\sigma^{\prime}(\omega)=\sigma_{0}+\sigma_{0} \cos (n \pi / 2)\left(\omega / \omega_{p}\right)^{n}, \\
\sigma^{\prime \prime}(\omega)=\sigma_{0} \sin (n \pi / 2)\left(\omega / \omega_{p}\right)^{n}+\omega \varepsilon_{\infty},
\end{gathered}
$$

so the real part of the permittivity $\varepsilon^{\prime}$ is

$$
\varepsilon^{\prime}(\omega)=\varepsilon_{\infty}+\left(\sigma_{0} / \omega_{p}\right) \sin (n \pi / 2)\left(\omega / \omega_{p}\right)^{n-1} .
$$

Lines in Figs. 3(a) and 3(b) are fits to the complex expression (8), from which the temperature dependence of the dc conductivity $\sigma_{0}$, the crossover frequency $\omega_{p}$ and the fractional exponent $n$ can be obtained as fitting parameters. Note that $\sigma_{0}$ is obtained from the low-frequency plateau of the real part of the conductivity. We want to emphasize the presence of the term $\cos (n \pi / 2)$ in expression (9a), which is often omitted $^{12,13}$ affecting the calculated value for the crossover frequency $\omega_{p}$.

The temperature dependence of the parameter $n$ is shown in Fig 4. A nearly constant value of 0.6 is obtained for the fractional exponent $n$ at temperatures above $470 \mathrm{~K}$. Below this temperature an increase in the slope of the conductivity towards $n=1$ is observed. We will focus now on the constant $n=0.6$ regime above $470 \mathrm{~K}$ to establish a connection with the time domain picture and the $n \approx 1$ regime will be discussed later.

The power-law regime is a description of the slowing down of the relaxation process as a result of cooperative effects, much in the same way as the KWW function does in 

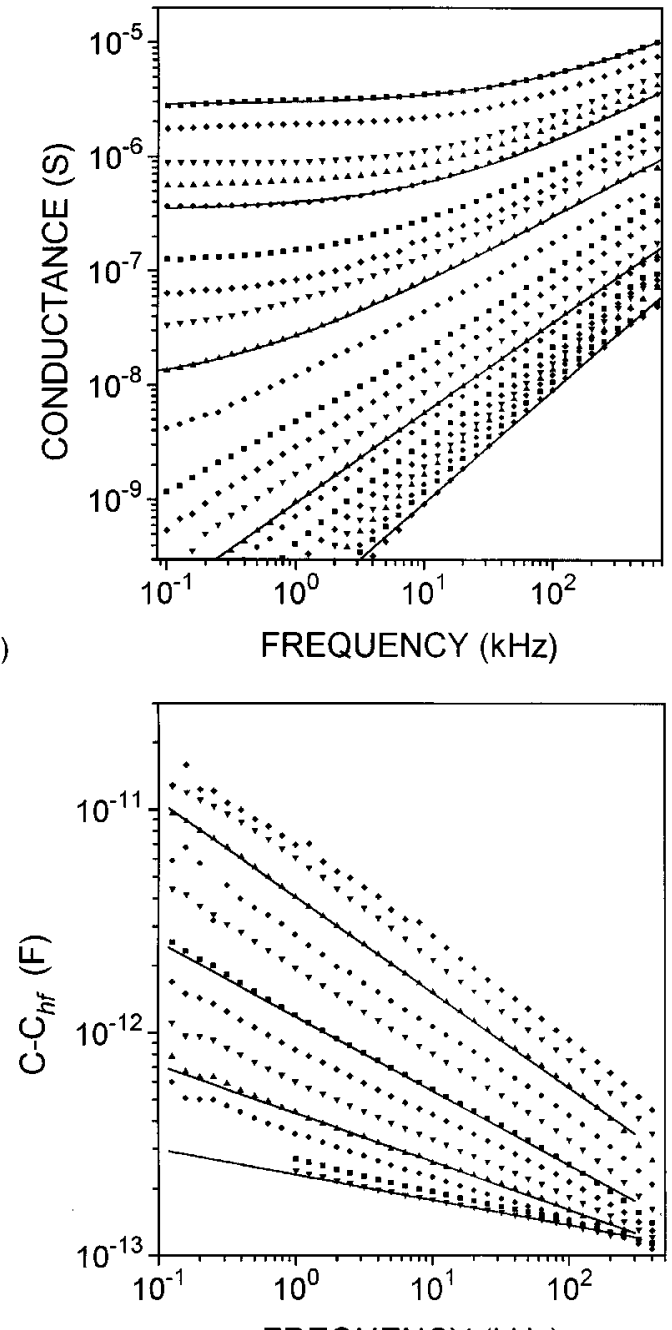

(b)

FREQUENCY $(\mathrm{kHz})$

FIG. 3. (a) Conductance vs frequency presented in a doublelogarithmic scale at several temperatures from 211 to $560 \mathrm{~K}$. Solid lines are fits to expression $\sigma^{\prime}(\omega)=\sigma_{0}+\sigma_{0} \cos (n \pi / 2)\left(\omega / \omega_{p}\right)^{n}$ for data at $211 \mathrm{~K}, 332 \mathrm{~K}, 450 \mathrm{~K}, 514 \mathrm{~K}$, and $560 \mathrm{~K}$. An increase in the exponent $n$ can be observed (slope of the curves at the higher frequencies), when temperature is decreased. (b) Capacitance data, from which the high-frequency value $C_{\mathrm{hf}}$ has been subtracted, are plotted vs frequency at several temperatures from 270 to $480 \mathrm{~K}$. Data are presented in a double-logarithmic scale to show their power-law dependence Solid lines are fits to expression $\varepsilon^{\prime}(\omega)-\varepsilon_{\infty}=\left(\sigma_{0} / \omega_{p}\right) \sin (n \pi / 2)\left(\omega / \omega_{p}\right)^{n-1}$ at $270 \mathrm{~K}, 332 \mathrm{~K}, 389$ $\mathrm{K}$, and $450 \mathrm{~K}$.

the time domain. However, as mentioned above, the connection between time and frequency domain formalisms involves the Fourier transform of the time derivative of the KWW decay function, and it is well known that there is no analytical expression for such Fourier transform. In this sense, both approaches cannot be regarded to be exactly equivalent. Since, on the other hand, both pictures are known to provide a good empirical description of the conductivity relaxation, there should be a connection between the parameters used in both descriptions. In fact, in the temperature range where modulus peaks were well resolved, $\beta$ values were approximately equal to $(1-n)$. We can also compare the characteristic time, $\tau_{p}$, obtained from the inverse of the

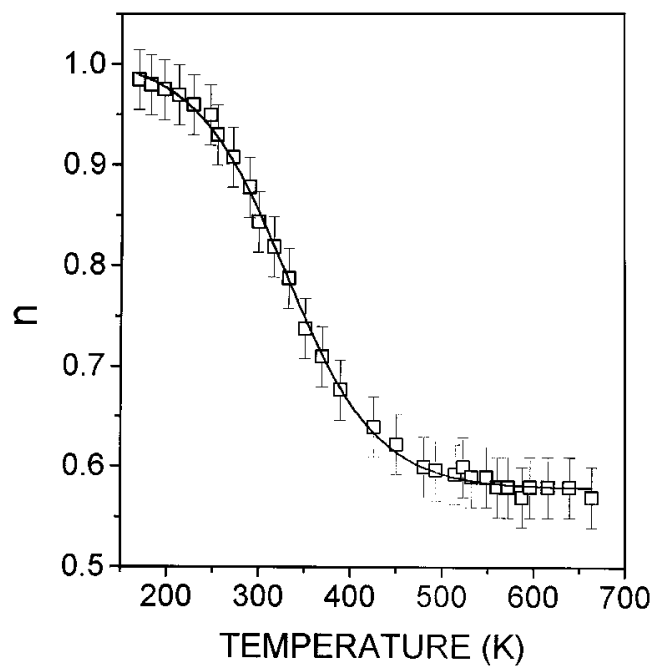

FIG. 4. Temperature dependence of the exponent $n$ obtained from conductivity vs frequency plots according to expression $\sigma^{\prime}(\omega)=\sigma_{0}+\sigma_{0} \cos (n \pi / 2)\left(\omega / \omega_{p}\right)^{n}$. The solid line is a guide for the eye.

crossover frequency $\omega_{p}$, with an average relaxation time $\langle\tau\rangle$ obtained from the KWW time decay function. The values obtained for $\langle\tau\rangle$ and the inverse of the crossover frequency $\left(\tau_{p}=1 / \omega_{p}\right)$ from expression (8) are plotted together in a logarithmic scale vs $1000 / T$ in Fig. 5. The line in the figure represents a thermally activated relaxation time of the form

$$
\tau=\tau_{\infty} \exp (E / k T)
$$

with $\tau_{\infty}=10^{-16} \mathrm{~s}$ and an activation energy $E=1.16 \mathrm{eV}$.

The excellent agreement between $\langle\tau\rangle$ and $\tau_{p}$ values seems to support the idea that they are actually the same time. This implies that the crossover frequency can be expressed as $\omega_{p}=\sigma_{0} / \varepsilon_{\infty}=1 /\langle\tau\rangle$. So, expression (8) may be rewritten as

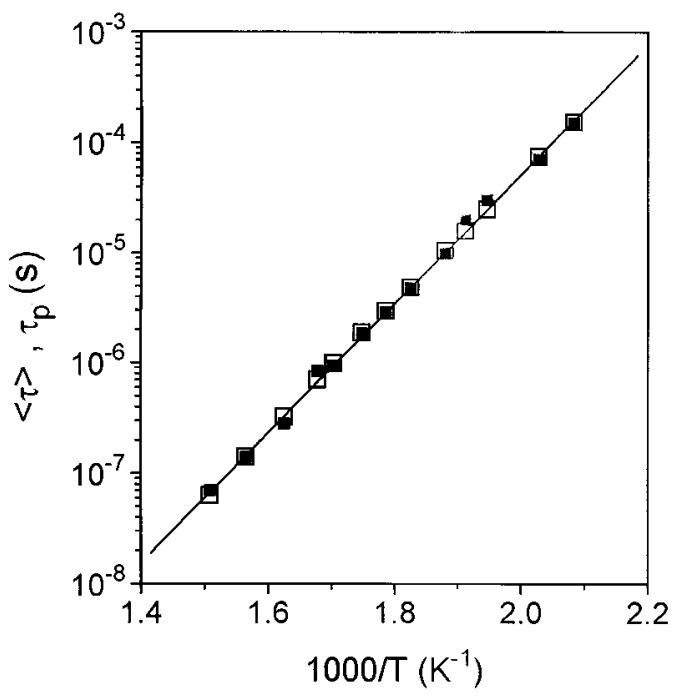

FIG. 5. Relaxation times $\langle\tau\rangle(\mathbf{\square})$ and $\tau_{p}(\square)$ vs $1000 / T$, from time and frequency domain analysis, respectively. Solid line is a fit from which a temperature activated relaxation time of the form $\tau=\tau_{\infty} \exp (E / k T)$ is obtained, with $\tau_{\infty}=10^{-16} \mathrm{~s}$ and activation energy $E=1.16 \mathrm{eV}$. 


$$
\sigma^{*}(\omega)=\sigma_{0}\left[1+\left(j \omega \frac{\varepsilon_{\infty}}{\sigma_{0}}\right)^{n}+j \omega \frac{\varepsilon_{\infty}}{\sigma_{0}}\right]
$$

This important result, which to the best of our knowledge is reported here for the first time, allows the dc conductivity to be obtained from the dispersive ac regime. Since $\varepsilon_{\infty}$ is directly obtained from experimental data, the dc conductivity, $\sigma_{0}$, can be obtained fitting expression (11) to the highfrequency power-law dependent conductivity. This result points to a common origin for both $\mathrm{dc}$ and ac regimes. Only at the lowest temperatures, when the value of $n$ changes towards 1 , the calculated dc conductivity becomes rather uncertain even for a small error in the estimated value of the parameter $n$.

Further information can be obtained using Ngai's coupling model. ${ }^{5}$ In this model the relaxation occurs independently for each ion at short times, and it is thermally activated with a microscopic activation energy $E_{a}$. At longer times, cooperative effects influence the relaxation giving rise to an increase in the effective energy barrier for ion motion up to a value $E$. The macroscopic activation energy, $E$, measured in the conductivity experiments is related to the microscopic one through $E=E_{a} / \beta$. Since values of $1.16 \mathrm{eV}$ and 0.43 were obtained for $E$ and $\beta$, respectively, a microscopic energy of $0.5 \mathrm{eV}$ can be deduced.

The ac conductivity, after expression (11), can be written as

$$
\sigma_{\mathrm{ac}}(\omega)=\sigma_{0}^{(1-n)} \cos (n \pi / 2)\left(\omega \varepsilon_{\infty}\right)^{n}
$$

It is therefore activated with $(1-n) E$, that is, with the microscopic activation energy $E_{a}$, since we have shown that $1-n \cong \beta$. The power-law increase of the conductivity towards high frequencies has been attributed to correlated ion motion. It has been proposed that correlation effects in the ion hopping conduction mechanism may force ions to backhop to their previous position after a hopping event. ${ }^{6}$ Therefore, the value of the conductivity depends on the measuring frequency: forward-backward hopping sequences within a time period $T$ do not contribute to the conductivity observed at frequencies lower than $1 / T$. They can be considered as unsuccessful hops in this time scale. Since most ions must be associated in this temperature range, and the ac conductivity is governed by the back hopping probability, it is reasonable to think of some relation between the microscopic energy and the association energy. In fact Greaves and $\mathrm{Ngai}^{7}$ have recently interpreted the microscopic energy $E_{a}$ in alkali silicate glassy ionic conductors in terms of an effective binding energy and a network conformational term which accompanies the hopping mechanism. Interestingly, our microscopic activation energy of $0.5 \mathrm{eV}$ is in good agreement with the $0.49 \mathrm{eV}$ proposed by Bauerle and $\mathrm{Hrizo}^{11}$ for the association energy, from dc conductivity measurements. It is also reasonably close to the values $0.6 \mathrm{eV}$ proposed for $\left[V_{\mathrm{O}} Y_{\mathrm{Zr}}^{\prime}\right]_{N N}$ and $\left[Y_{\mathrm{Zr}}^{\prime} V_{\mathrm{O}} Y_{\mathrm{Zr}}^{\prime}\right]^{X}$ clusters from theoretical calculations. ${ }^{14,15}$ This result may give a clue to understand correlations in these materials in terms of the role played by the environment. Ultimately, it points to the association mechanism as responsible for the increased back hopping probability, and consequently for the slowing down of the relaxation process.

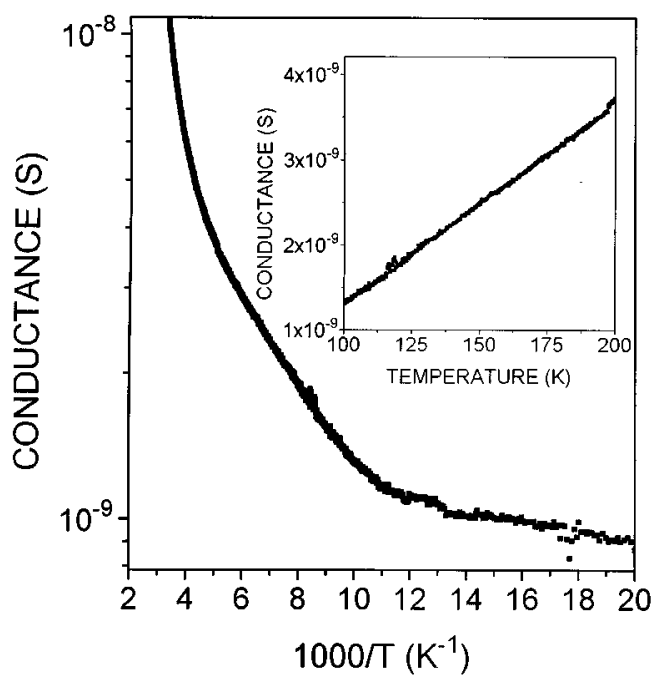

FIG. 6. Temperature dependence of conductance at $30 \mathrm{kHz}$ in an Arrhenius fashion. The inset shows the linear dependence on temperature between 100 and $200 \mathrm{~K}$.

Concerning the $n \approx 1$ regime in the ac conductivity, it has been already observed by other authors, and referred to as a " "new universality.", 16 Some typical crystalline ionic conductors like $\mathrm{RbAg}_{4} \mathrm{I}_{5}$ (Ref. 17) are known to exhibit power-law exponents smaller than unity over wide frequency and temperature ranges merging into a high-frequency plateau, and not showing the $n \approx 1$ regime. In others, like $\mathrm{Na}-\beta-\mathrm{Al}_{2} \mathrm{O}_{3},{ }^{18}$ this regime shows up at sufficiently low temperature. Glassy materials, on the other hand, usually show this non-Jonscher linear frequency dependence of the conductivity and, in this sense, it has been recently reported to be a distinctive feature of glassy materials. ${ }^{19}$ It is clear from the conductivity data in Fig. 3 that the $n \approx 1$ term is present in single crystal YSZ. Moreover a suppression of the thermal activation of the ac conductivity is observed when the temperature is lowered below $250 \mathrm{~K}$, which has also been reported to occur in glassy materials. Figure 6 shows the temperature dependence of the ac conductivity at $30 \mathrm{kHz}$ in an Arrhenius fashion. A very small activation energy can be observed between 100 and $200 \mathrm{~K}$, which yields linear conductivity vs temperature plots in a linear scale (also shown in Fig. 6 as an inset). The reduction in the activation energy when $n$ tends to one can be understood in view of expression (12). Small activation energies have been interpreted in terms of localized hopping in a nonperiodic potential ${ }^{20}$ arising from disordered distribution of ions. A model has been proposed for inorganic glasses, ${ }^{21}$ which explains the low temperature $(T<200 \mathrm{~K})$ behavior of the nuclear spin relaxation in terms of thermally activated low-frequency excitations of disordered modes. The modes are described by asymmetric double-well potential configurations (ADWP) with suitable densities of states. This picture probably applies to the crystalline YSZ in the sense that in the associated regime at low temperatures, perturbations of the periodic potential will arise from the nonequivalence of $\mathrm{Y}$ and $\mathrm{Zr}$ sites, and may be also from the mutual interaction of randomly distributed oxygen vacancies. This may be in connection with the glasslike properties observed in doped group IV $A$ ion conducting oxides with fluorite structure at low tempera- 
tures $(1-40 \mathrm{~K}){ }^{22}$ Localization applies here in the sense of oxygen vacancies linked to the Yttrium sites may be performing strongly correlated $(n \cong 1)$, dipolelike oscillations. In fact localized excitations ascribed to oxygen vacancies have been reported from Raman and specific heat measurements. ${ }^{23}$

In summary we have presented a study on the effect of correlated ion motion on the electrical conductivity relaxation in single-crystalline yttria-stabilized zirconia. An analysis of the frequency dependence of electric modulus has allowed us to obtain an analytical distribution of relaxation times which allows the calculation of the time decay function by numerical integration. Time decay functions, $\Phi(t)=\exp \left[-\left(t / \tau^{*}\right)^{\beta}\right]$, of the Kohlrausch-Williams-Watts kind has been obtained, similar to those frequently found in glassy ionic conductors. Dispersive conductivities in the frequency domain of the form $\sigma^{*}(\omega)=\sigma_{0}+\sigma_{0}\left(j \omega / \omega_{p}\right)^{n}$ $+j \omega \varepsilon_{\infty}$ have been also found. The relaxation parameters $\beta$ and an average relaxation time $\langle\tau\rangle$ has been compared with the inverse of the crossover frequency $\tau_{p}=1 / \omega_{p}$ and $1-n$, showing very good agreement, such that we have been able to show that $\omega_{p}=\sigma_{0} / \varepsilon_{\infty}$. This points to a common origin for ac and dc processes, as reflected by the following expression for the complex conductivity

$$
\sigma^{*}(\omega)=\sigma_{0}\left[1+\left(j \omega \frac{\varepsilon_{\infty}}{\sigma_{0}}\right)^{n}+j \omega \frac{\varepsilon_{\infty}}{\sigma_{0}}\right] .
$$

A single-ion microscopic activation energy of $0.5 \mathrm{eV}$ has been obtained following Ngai's coupling model which has been related to the association energy for oxygen vacancies. A regime with $n \approx 1$ has been found at low temperatures, which appears often in glassy ionic conductors, and it has been associated with localized hopping of oxygen vacancies performing dipolelike oscillations.
${ }^{1}$ See the review by T. H. Etsell and S. N. Flengas. Chem. Rev. 70, 339 (1970).

${ }^{2}$ J. D. Solier, I. Cachadiña, and A. Dominguez-Rodriguez, Phys. Rev. B 48, 3704 (1993).

${ }^{3}$ A. K. Jonscher, Dielectric Relaxation in Solids (Chelsea Dielectric, London, 1983).

${ }^{4}$ R. Kohlrausch, Ann. Phys. (Leipzig) 72, 393 (1847).

${ }^{5}$ K. L. Ngai, Comments Solid State Phys. 9. 121 (1979); 9, 141 (1980); for a recent review, see K. L. Ngai, in Effects of Disorder on Relaxational Processes, edited by R. Richert and A. Blumen (Springer-Verlag, Berlin, 1994), p. 89.

${ }^{6} \mathrm{~K}$. Funke, in Superionic Solids and Solid Electrolytes: Recent Trends, edited by A. L. Laskar and S. Chandra (Academic, New York, 1989); Prog. Solid State Chem. 22, 111 (1993).

${ }^{7}$ G. N. Greaves and K. L. Ngai, Phys. Rev. B 52, 6358 (1995).

${ }^{8}$ S. Havriliak and S. Nagami, Polymer 8, 161 (1967).

${ }^{9}$ J. Colmenero, A. Alegria, J. M. Alberdi, F. Alvarez, and B Frick, Phys. Rev. B 44, 7321 (1991).

${ }^{10}$ K. L. Ngai, R. W. Rendell, and H. Jain, Phys. Rev. B 30, 2133 (1984).

${ }^{11}$ J. E. Bauerle and J. Hrizo, J. Phys. Chem. Solids 30, 565 (1969).
${ }^{12}$ D. P. Almond, G. K. Duncan, and A. R. West, Solid State Ion. 8, 159 (1983).

${ }^{13}$ D. P. Almond and A. R. West, Solid State Ion. 9/10, 277 (1983).

${ }^{14}$ V. Butler, C. R. A. Catlow, and B. E. F. Fender, Solid State Ion. 5, 1 (1981).

${ }^{15}$ D. Bingham, P. W. Tasker, and A. N. Cormack, Philos. Mag. A 60, 1 (1989).

${ }^{16}$ W. K. Lee, J. F. Liu, and A. S. Nowick, Phys. Rev. Lett. 67, 1559 (1991).

${ }^{17}$ K. Funke, Prog. Solid State Chem. 22, 111 (1993).

${ }^{18}$ U. Strom, K. L. Ngai, and O. Kanert, J. Non-Cryst. Solids 131133, 1011 (1991).

${ }^{19}$ C. Cramer, K. Funke, and T. Saatkamp, Philos. Mag. B 71, 701 (1995).

${ }^{20}$ W. Dieterich, D. Knödler, and P. Penzig, J. Non-Cryst. Solids 172-174, 1237 (1994).

${ }^{21}$ O. Kanert, J. Steinert, H. Jain, and K. L. Ngai, J. Non-Cryst. Solids 130, 1001 (1991).

${ }^{22}$ D. A. Ackerman, D. Moy, R. C. Potter, A. C. Anderson, and W. N. Lawless, Phys. Rev. B 23, 3886 (1981).

${ }^{23}$ W. N. Lawless, Phys. Rev. B 21, 585 (1980). 